\title{
Radical Fragment Ions in Collision-Induced Dissociation Mass Spectrometry
}

2

\author{
Shipei Xing ${ }^{1}$, Tao Huan ${ }^{1, *}$
}

4

$5 \quad{ }^{1}$ Department of Chemistry, Faculty of Science, University of British Columbia, Vancouver

6 Campus, 2036 Main Mall, Vancouver, V6T 1Z1, BC, Canada

$10 *$ Author to whom correspondence should be addressed:

11

12 Dr. Tao Huan

13 Tel: (+1)-604-822-4891

14 E-mail: thuan@chem.ubc.ca

15 Website: https://huan.chem.ubc.ca/

16

17 
19 Collision-induced dissociation (CID) is a common fragmentation strategy in mass spectrometry

20 (MS) analysis. A conventional understanding is that fragment ions generated in low-energy CID

21 should follow the even-electron rule. As such, (de)protonated precursor ions should predominately

22 generate (de)protonated fragment ions with very few radical fragment ions (RFIs). However, the

23 extent to which RFIs present in $\mathrm{MS}^{2}$ spectra has not been comprehensively investigated. This work

24 uses the latest NIST 20 tandem mass spectral library to investigate of the occurrence of RFIs in

25 CID $\mathrm{MS}^{2}$ experiments. In particular, RFIs were recognized using their integer double bond

26 equivalent (DBE) values calculated from their annotated molecular formulas. Our study shows

27 unexpected results as $65.4 \%$ and $68.8 \%$ of $\mathrm{MS}^{2}$ spectra contain at least $10 \%$ RFIs by ion-count

28 (total number of ions) in positive and negative electrospray ionization (ESI) modes, respectively.

29 Furthermore, we classified chemicals based on their compound classes and chemical substructures,

30 and calculated the percentages of RFIs in each class. Results show that "Organic 1,3-dipolar

31 compounds" and "Lignans, neolignans and related compounds" are the top 2 compound

32 superclasses which tend to produce RFIs in their CID $\mathrm{MS}^{2}$ spectra. Moreover, aromatic,

33 arylbromide, heteroaromatic, alkylarylether, phenol, and conjugated double bond-containing

34 chemicals are more likely to produce RFIs. We also found four possible patterns of change in RFI

35 percentages as a function of CID collision energy. Finally, we demonstrate that the inadequate

36 consideration of RFIs in most conventional bioinformatic tools might cause problems during in

37 silico fragmentation and de novo annotation of $\mathrm{MS}^{2}$ spectra. This work provides a further

38 understanding of CID $\mathrm{MS}^{2}$ mechanism, and the unexpectedly large percentage of RFIs suggests a

39 need for consideration in the development of bioinformatic software for $\mathrm{MS}^{2}$ interpretation. 


\section{Introduction}

42 Collision-induced dissociation (CID) is a common ion activation technique used in mass 43 spectrometry (MS) analysis to generate tandem MS (MS') spectra for chemical structure 44 determination.[1-5] The CID process generates fragment ions to obtain a fragment ion spectrum.

45 During the CID event, heterolytic fragmentation generates (de)protonated fragment ions and

46 homolytic fragmentation generates radical fragment ions (RFIs). The CID collision energy is a 47 laboratory frame collision energy, and the center of mass energy slightly varies for different 48 precursor ions depending on their masses. In low-energy CID (energy less than $100 \mathrm{eV}$ ) used in 49 MS-based chemical and biochemical analyses, it is commonly believed that CID predominantly 50 generates fragmentation of protonated or deprotonated species. In comparison, RFIs are 51 energetically not favorable and thus are rare. Another common belief is that RFIs are generated 52 because there is a radical cation or anion precursor as the consequence of applying a high voltage 53 during electrospray ionization (ESI). Besides several reports on RFIs in some targeted chemical 54 classes,[6] the global investigation on the percentage of RFIs in CID has not been systematically 55 studied.

57 With the development of high-resolution liquid chromatography-mass spectrometry (LC-MS) 58 systems, it is now possible to achieve a comprehensive and untargeted coverage of chemical 59 species in a biological or environmental sample. The application of CID then becomes critical to 60 generate $\mathrm{MS}^{2}$ spectra for chemical annotation.[7-9] In particular, due to the large volume of 61 chemical signals detected in experiments and a limited number of chemical standards in $\mathrm{MS}^{2}$ 62 spectral libraries, de novo interpretation and in silico prediction of $\mathrm{MS}^{2}$ spectra from chemical 63 structures have become important.[10, 11] In the development of above-mentioned $\mathrm{MS}^{2}$ 
64 interpretation programs, it is important to have a clear understanding of fragmentation mechanisms

65 in order to develop powerful and robust bioinformatic tools. Conventionally, it is thought that since

66 ESI produces even-electron species and the fragmentation method is of relatively low energy, CID

67 should generate even-electron species almost exclusively as well - the chance of generating RFIs

68 is exceedingly rare. However, to the best of our knowledge, there is no comprehensive study of

69 the types of fragment ions generated in CID MS ${ }^{2}$ at a global scale.

71 In this work, we studied the existence of RFIs in CID MS ${ }^{2}$ spectra using the NIST 20 high-

72 resolution $\mathrm{MS}^{2}$ spectral library (https://www.nist.gov/srd/nist-special-database-20), hereafter

73 referred to as NIST 20. The NIST 20 contains 1,026,717 $\mathrm{MS}^{2}$ spectra for 27,613 unique chemical

74 compounds (positive ion mode: 765,385 spectra for 26,600 chemicals; negative ion mode: 261,332

75 spectra for 11,675 chemicals). One important feature of NIST 20 is that fragment ions have been

76 annotated with molecular formulas. Using the molecular formula information, we can calculate a

77 double bond equivalent (DBE) value for each fragment ion. Since RFIs do not follow even-electron

78 rules, their DBE values are integers. Using this information, we can determine whether an

79 annotated fragment ion in NIST 20 is an RFI or not. The RFI information of all the chemicals in

80 NIST 20 was then used for a comprehensive investigation, including (1) calculating the ion-count

81 (total number of ions) and ion-intensity (total ion intensity) percentages of RFIs and plotting their

82 distributions; (2) categorizing chemicals by their ontology classes and checking class-specific and

83 substructure-specific RFI distributions; (3) investigating the relationship between RFIs and CID

84 collision energy; and (4) summarizing the potential problems of not including RFIs in in silico

$85 \mathrm{MS}^{2}$ generation and de novo $\mathrm{MS}^{2}$ interpretation. This work represents a systematic and holistic 
86 study of RFIs in CID MS ${ }^{2}$ spectra, providing guidance for the future development of bioinformatic 87 tools for $\mathrm{MS}^{2}$ interpretation.

88 


\section{Methods}

Pretreatment of NIST 20 Tandem MS Spectral Library. NIST 20 was purchased from NIST

91 through Isomass Scientific Inc. NIST 20 contains a total of 1,026,717 low-energy CID MS ${ }^{2}$ spectra

92 for 27,613 unique chemical compounds. It includes 765,385 spectra for 26,600 chemicals in

93 positive ion mode and 261,332 spectra for 11,675 chemicals for negative ion mode. These high

94 resolution $\mathrm{MS}^{2}$ spectra were collected from Thermo Orbitrap mass spectrometers. More than $99.5 \%$

95 of the $\mathrm{MS}^{2}$ spectra were obtained using nitrogen as the collision gas, while others used helium.

96 Molecular formula annotation of all fragment ions was completed using MS Interpreter, a

97 bioinformatic tool embedded in the NIST MS Search program. The detailed explanation of how

98 NIST MS Search performs subformula annotation can be found in Text S1.

To prepare NIST 20 for the study, we first removed $\mathrm{MS}^{2}$ spectra of uncommon precursor ions,

101 such as the isotopic peak(s) of a precursor (e.g., $\mathrm{M}+1, \mathrm{M}+2$ ) and doubly and triply charged

102 adducts (e.g., $[\mathrm{M}+\mathrm{Na}+\mathrm{H}]^{2+}$ ). We also discarded $\mathrm{MS}^{2}$ spectra with fewer than 5 annotated

103 fragments. Furthermore, $\mathrm{MS}^{2}$ spectra with radical precursor ions (Figures S1 \& S2) were removed

104 to ensure that all RFIs were generated from (de)protonated (or even-electron) precursor ions. When

105 multiple $\mathrm{MS}^{2}$ spectra were available for a given chemical compound, the $\mathrm{MS}^{2}$ spectrum with the

106 most fragment ions was used for further interpretation. It is important to note that not all fragment

107 ions in NIST 20 have molecular formula annotations. Overall, $88.4 \%$ and $87.0 \%$ of the fragment

108 ions are annotated in positive and negative ion modes, respectively. For a fragment with multiple

109 annotations, only the smallest mass error one was kept.

111 Analysis of NIST 20. Data analysis was conducted using R language (version 4.0.3). The R 112 package $C H N O S Z$ (version 1.4.0) was used to parse and write molecular formulas. RFIs were 
113 determined via the annotated subformula information. More specifically, double bond equivalent

114 (DBE) values were calculated for given subformulas using the equation shown below. Letters

115 represent the number of each chemical element in a molecular formula.

$$
D B E=C+S i+1-\frac{H+F+C l+B r+I+N a+K}{2}+\frac{N+P}{2}
$$

117 Following the LEWIS rule that electrons in main group element-based molecules are shared such

118 that s- and p-valence shells of all atoms are fully filled, fragment ions with non-integer DBE values

119 are (de)protonated ions and fragment ions with integer DBE values are RFIs.

121 To study the relationship between RFIs and compound classes, chemical compounds were first 122 systematically classified using ClassyFire[12] (Tables $\mathbf{S 1}$ for positive ion mode results and $\mathbf{S 2}$ for 123 negative ion mode results). In brief, the InChIKey, a textual identifier for chemical substances, of 124 each chemical in NIST 20 was used as an input for the function "get_classification" from the 125 classyfireR package (version 0.3.6). The "get_classification" function assigned hierarchical 126 classification results for each chemical, and the class levels of "superclass", "class", and "subclass" 127 defined in ClassyFire[13] were used for further analysis. Moreover, only superclasses containing 128 more than $0.1 \%$ of the total compounds were kept.

130 The relationship between chemical substructures and RFI percentages were investigated using the 131 R package $r c d k$ (version 3.5.0). The $\mathrm{R}$ package contains a total of 307 substructures from 132 Chemistry Development Kit (CDK).[14] The entire CDK substructure list can be found in Table 133 S3. To recognize chemical substructures, the InChIKey of each chemical compound was converted 134 to a SMILES string using the PubChem Identifier Exchange Service platform 
135 (https://pubchem.ncbi.nlm.nih.gov/idexchange). The SMILES string of a chemical is then used to

136 get all possible fingerprint(s) in that structure using the function "get.fingerprint" from $r c d k$.

138 To understand the patterns of how RFI count and intensity percentages change as a function of 139 collision energy, an algorithm was created. We first prepared an RFI percentage vector sorted by 140 collision energy in ascending order. Then, we split the vector into two halves. For each half, 141 Spearman correlation is calculated between the order of collision energy and the RFI percentages $142\left(\mathrm{X}_{\mathrm{i}}\right)$. After both Cor1 (the first half) and Cor2 (the second half) were calculated, the RFI pattern 143 (e.g., pattern I, II, III, or IV) was determined using the following decision table:

\section{4}

\begin{tabular}{|l|c|c|}
\hline Pattern & Cor $_{1} \geq 0$ & Cor $_{1}<0$ \\
\hline Cor $_{2} \geq 0$ & I & II \\
\hline Cor $_{2}<0$ & III & IV \\
\hline
\end{tabular}

\section{Implications of RFIs in De Novo Annotation}

146 To demonstrate the limited capacity of annotating RFIs in state-of-the-art bioinformatics tools, we

147 tested NIST $20 \mathrm{MS}^{2}$ spectra using SIRIUS 4[15], one of the most commonly used $\mathrm{MS}^{2}$

148 interpretation software. We randomly sampled 1000 RFI-containing $\mathrm{MS}^{2}$ spectra from NIST 20

149 (500 per ionization mode) using their integer DBE values. These $\mathrm{MS}^{2}$ spectra were then imported 150 into SIRIUS 4 and subjected to molecular formula prediction and fragmentation tree calculation

151 (see Text S2 for the detailed SIRIUS 4 parameters). For all fragment ions interpreted by SIRIUS

1524 , their molecular formulas were used to determine whether they were (de)protonated ions or RFIs.

153 These SIRIUS annotation results were then compared to the NIST annotated subformulas to 154 calculate RFI annotation sensitivity (i.e., the fraction of RFIs correctly annotated by SIRIUS 4). 


\section{Results and Discussion}

\section{Radical Fragment Ions in NIST 20}

158 A total of 765,385 spectra for 26,600 chemicals in positive ion mode and 261,332 spectra for

15911,675 chemicals for negative ion mode were collected from NIST 20. After removing disqualified

$160 \mathrm{MS}^{2}$ spectra, including spectra with radical precursor ions, multiple-charged adducts, and fewer 161 than 5 annotated fragments, a total of $470,841 \mathrm{MS}^{2}$ spectra for 24,140 chemicals in positive ion 162 mode and $137,308 \mathrm{MS}^{2}$ spectra for 9,764 chemicals in negative ion mode were used for the 163 following studies. It was interesting to find that 11.5 and $14.3 \%$ of the $\mathrm{MS}^{2}$ spectra in positive and 164 negative ion modes contained radical precursor ions, respectively (Figure S2). In addition, over $16570 \%$ of the $\mathrm{MS}^{2}$ spectra had at least 5 annotated fragments. The distributions of annotated $\mathrm{MS}^{2}$ 166 spectra fragments are presented in Figure S3.

168 Figure 1 illustrates the schematic workflow of investigating RFIs in NIST $20 \mathrm{MS}^{2}$ spectra. We 169 first calculated the percentages of RFIs and (de)protonated ions in each NIST $20 \mathrm{MS}^{2}$ spectrum 170 (Tables S4 \& S5) and plotted their distributions. In particular, distributions of both ion-count and 171 ion-intensity percentages were plotted throughout this work to gain a more comprehensive view 172 of RFIs in $\mathrm{MS}^{2}$ spectra. Figures $2 \mathrm{~A}$ and $\mathbf{2 C}$ show the results of NIST $20 \mathrm{MS}^{2}$ spectra in positive 173 and negative ion modes, respectively. Here we consider $\mathrm{MS}^{2}$ spectra with $\leqslant 10 \%$ RFIs as low174 RFI and $>10 \%$ RFIs as high-RFI $\mathrm{MS}^{2}$ spectra. In the positive ion mode $\mathrm{MS}^{2}$ spectra, $34.6 \%$ $175\left(162,746\right.$ out of 470,841) are low-RFI and $65.4 \%(308,095$ out of 470,841$)$ are high-RFI MS ${ }^{2}$ 176 spectra. Similar results were also found in the negative ion mode $\mathrm{MS}^{2}$ spectra, as $31.2 \%(42,798$ 177 out of 137,308$)$ are low-RFI and $68.8 \%(94,510$ out of 137,308$)$ are high-RFI MS ${ }^{2}$ spectra. The 
178 results of these ion-count percentages were unanticipated, given the common belief that RFIs are

179 very rare in low-energy CID $\mathrm{MS}^{2}$ spectra.

180

181 Besides the ion-count percentages, we also studied the ion-intensity percentages of RFIs in both 182 positive and negative ion modes (Tables S6 \& S7). As shown in Figures 2B and 2D, for 74.2\% $183(349,163$ out of 470,841$)$ of positive ion mode and $71.3 \%(97,930$ out of 137,308$)$ of negative ion 184 mode $\mathrm{MS}^{2}$ spectra, RFIs only account for less than $20 \%$ of the total ion intensities. A comparison 185 to ion-count percentages clearly shows that although an unexpectedly high number of RFIs are 186 found in $\mathrm{MS}^{2}$ spectra, their ion intensities are relatively low. This might be related to their low 187 chemical stability compared to (de)protonated ions.

\section{Radical Fragment Ions and Their Precursor Compound Classes}

190 To further understand which chemical compounds are more likely to generate RFIs in CID MS ${ }^{2}$ 191 experiments, we calculated both ion-count and ion-intensity percentages of RFIs and classified the 192 corresponding chemical compounds using ClassyFire[13] on three class levels, including 193 "superclass", “class", and "subclass". At the superclass level, for all 22,756 compounds in positive 194 ion mode and 8,764 compounds in negative ion mode, 17 superclasses were assigned. Figure 3A 195 shows the RFI count percentage distributions of the superclasses by descending median values 196 (superclasses containing more than $0.1 \%$ of the total compounds were plotted here, 13 superclasses

197 for each ion mode). As we can see from Figure 3A, the overall median RFI count percentage is $19827.3 \%$ for positive ion mode and $21.2 \%$ for negative ion mode. Compound superclasses with RFI 199 percentage medians larger than the overall median ("All" in the plot) were labelled in red and RFI 200 percentage medians smaller than the overall median in blue. In both positive and negative ion 
201 modes, "Organic 1,3-dipolar compounds" and "Lignans, neolignans and related compounds" are

202 the top 2 compound superclasses and tend to produce RFIs in their CID MS ${ }^{2}$ spectra. This can be

203 attributed to their abundant conjugated $\pi$-bond systems, which help to stabilize RFIs with

204 delocalized electrons. On the other side, RFIs are rarely found in $\mathrm{MS}^{2}$ spectra of superclasses

205 "Lipids and lipid-like molecules" and "Organic acids and derivatives". This result agrees with our

206 conventional understanding that compounds with long carbon chains are generally not preferable

207 for RFIs compared to conjugated systems. Similar trends can be obtained using the distributions

208 of RFI intensity percentages as shown in Figure S4.

209

210 Next, we generated sunburst plots of RFI percentage distributions in terms of the three levels of 211 compound classes in both polarity modes. Figure 3B illustrates the sunburst plot of RFI count

212 percentage in positive ion mode. The RFI count percentages of all compound classes at different

213 class levels can be found detailed in Table S8. As we can see in Figure 3B, slices from the inner

214 layer to the outer layer represent compound class levels of "superclass", "class", and "subclass".

215 The median RFI count percentage in each class was calculated, and their corresponding class

216 blocks in Figure 3A were distinguished by color, where dark red denotes RFI count percentage

217 higher than median and dark blue denotes lower than median. Interestingly, various compound

218 classes that belong to the same superclass can behave substantially different from each other. For

219 instance, both "Fatty acyls" and "Steroids and steroid derivatives" have the superclass "Lipids and

220 lipid-like molecules", but the median RFI count percentage of "Fatty acyls" is only $1.2 \%$ and much

221 smaller compared to the $17.9 \%$ of "Steroids and steroid derivatives". The fused ring system of

222 steroid molecules render them more inclined to RFIs during the CID process. Similarly, the

223 "Naphthacenes" class (37.7\%) has higher RFI count percentage than "Benzene and substituted 
224 derivatives" (31.2\%), even though they are of the same superclass "Benzenoids". As

225 "Naphthacenes" are four-ringed chemicals of polycyclic aromatic hydrocarbons, it is apparent that

226 compounds with larger conjugated electron systems have higher RFI percentages. Similarly, the

227 RFI count percentage in negative mode results are shown in Figure S5 and Table S9. Moreover,

228 we also generated sunburst plots and result tables using the ion-intensity percentages of RFIs.

229 Relevant results can be found in Figures S6-S7 and Tables S10-S11. These informative plots 230 provide comprehensive knowledge of RFIs in the CID $\mathrm{MS}^{2}$ spectra of various chemical classes.

232 Radical Fragment Ions and Chemical Substructures

233 Furthermore, we investigated which chemical substructure is more likely to lead to RFI generation 234 in CID $\mathrm{MS}^{2}$ events. In this study, a CDK substructure system containing 307 chemical 235 substructures (Table S3) was selected. In total, 23,478 unique chemicals in positive ion mode 236 (Table S12) and 9,411 unique chemicals in negative ion mode (Table S13) were successfully 237 assigned with at least one CDK substructure. For each chemical substructure, we categorized all 238 the chemical compounds into two groups based on the compound containing or not containing that 239 specific chemical substructure. We then performed Mann-Whitney U test, a nonparametric test to 240 determine statistical significance, between the RFI percentages (both ion-count and ion-intensity) 241 of the two classes. Statistical results of positive and negative ion modes are tabulated in Tables $242 \mathbf{S 1 4}$ and S15, respectively. Out of the 307 total substructures, 127 substructures have $P$ values of 243 less than 0.01 based on RFI count percentage in positive ion mode. Chemicals that contain any of 244 these 127 substructures have significantly different RFI count percentages than those that do not. 245 Of the 127 substructures, 65 have significantly higher RFI count percentages in the substructure246 containing chemicals, suggesting that chemicals containing these substructures are more likely to 
247 generate RFIs. In Figure 4A, we showcase four representative substructures that have the highest

248 statistical significance $(P<1 \mathrm{e}-3)$. It can be clearly seen that all of these chemical substructures

249 have conjugated $\pi$-bond systems, which contributed to their significantly higher RFI count

250 percentages.

251

252 We also performed a similar analysis to all the compounds in negative ion mode. Negative ion 253 mode analysis results show 94 substructures with $P$ values of less than 0.01 . Among them, 46

254 substructures lead to more RFI generation when a chemical contains it. Four of the top-ranked 255 substructures, including arylfluoride, arylchloride, arylbromide and aryliodide, are shown in

256 Figure 4B. The detailed results can be found in Table S15. Overall, the aromatic substructure 257 consistently leads to more RFIs in both positive and negative ion modes.

259 Intensity of Radical Fragment Ions and CID Collision Energy

260 Furthermore, we tried to understand how the change of CID collision energy affects the production

261 of RFIs. Our conventional understanding is that higher CID collision energy is more likely to 262 generate RFIs. In this work, we investigated the correlation between RFI intensities and CID 263 collision energies using the chemicals in NIST 20. An important feature of NIST 20 is that it 264 provides $\mathrm{MS}^{2}$ spectra collected from up to 24 different collision energies. We calculated RFI 265 intensity percentages from $\mathrm{MS}^{2}$ spectra at each collision energy and checked the change as a 266 function of collision energy. After manually checking dozens of chemicals, we summarized four 267 possible patterns as shown in Figure 5A. Type I, in which the percentage of RFI intensities keeps 268 increasing with the increase of collision energy, is the most common. Interestingly, there are three 
269 other types of RFI intensity percentage change; Type II, decreases and then increases; Type III,

270 increases and then decreases; and Type IV, keeps decreasing.

271

272 We then automatically determined the type of RFI percentage for all 24,140 and 9,764 chemicals

273 in positive and negative ionization modes, respectively, as $\mathrm{MS}^{2}$ spectra at multiple collision

274 energies were available. As shown in Figure 5B, most chemical compounds generate RFI

275 percentages of Type 1, which account for $61.0 \%$ in positive ion mode and $40.5 \%$ in negative ion

276 mode. An interpretation for the chemicals belonging to Type I is that most of their RFIs are of

277 small structural pieces at the bottom leaves of fragmentation trees[16], and thus they are inclined

278 to be produced under higher collision energies. As an example, we manually interpreted a

279 fragmentation pathway for the $\mathrm{MS}^{2}$ spectrum of lithocholic acid (Figure S9). All the RFIs of

280 lithocholic acid are the end products of the fragmentation pathway. Therefore, their intensities

281 keep increasing with the increased collision energies. Conversely, Type IV RFI intensity

282 percentages, those that decrease with collision energy, usually happens when the RFIs show up at

283 the root branches of fragmentation trees. Although not very common, Type IV RFIs account for

$2849.8 \%$ in positive ion mode and $20.0 \%$ in negative ion mode. On the other side, Type II and Type

285 III are more complicated. It is possible that in these two cases, RFIs show up at different positions

286 in the fragmentation pathways.

287

288 Apart from RFI intensity percentages, we also looked into the distribution and patterns of RFIs as

289 a function of collision energy using RFI count percentage in both polarity modes (see Figure S8).

290 Likewise, Type I is the most common, accounting for $57.6 \%$ and $30.6 \%$ in positive and negative

291 ion modes, respectively. The results above show that instead of being positively correlated with 
292 collision energy, the pattern of RFIs varies and depends on the position of the RFI in the 293 fragmentation pathway.

\section{Potential Issues of Not Considering Radical Fragment Ions}

296 A clear understanding of $\mathrm{MS}^{2}$ spectra is critical to its interpretation in chemical annotation and 297 unknown identification.[17] Currently, RFIs in $\mathrm{MS}^{2}$ spectra are usually ignored during the process

298 of untargeted metabolomics data. This leads to incomplete in silico predicted fragment ions in $\mathrm{MS}^{2}$ 299 spectra as well as missing or incorrect annotations of true RFIs in experimental MS ${ }^{2}$ spectra. To 300 understand this, we first summarized some well-established bioinformatic software that perform 301 in silico fragmentation for unknown identification (Table 1). It can be clearly seen in the table that 302 the majority of the software have not fully considered the existence of RFIs. To minimize the 303 amount of false positive fragments as well as improve the computational speed, even-electron rules 304 are usually applied while neglecting RFIs during the in silico prediction process. Given the 305 considerable percentage of RFIs in our NIST 20 study, we believe that the incorporation of RFIs 306 in the development of in silico $\mathrm{MS}^{2}$ generation can significantly boost their performance.

308 Next, we demonstrated the limited RFI annotation of current bioinformatics tools using SIRIUS $3094[15]$, which is one of the commonly used $\mathrm{MS}^{2}$ interpretation software. By randomly sampling 3101000 NIST $20 \mathrm{MS}^{2}$ spectra containing RFIs (500 per ionization mode) and comparing the 311 annotated RFIs against NIST annotation, the distribution plots of RFI annotation sensitivity are 312 shown in Figures 6A and $\mathbf{6 C}$ for positive and negative ion modes, respectively. In general, $47.4 \%$ 313 of the positively ionized $\mathrm{MS}^{2}$ spectra and $57.8 \%$ of the negatively ionized $\mathrm{MS}^{2}$ spectra have lower 314 than $10 \%$ RFI annotation sensitivity. This low annotation sensitivity suggests that most RFIs 
315 remain poorly annotated by SIRIUS. However, considering that the intrinsic design of SIRIUS 4

316 allows only a few common radical losses[18], this result can be expected. To further explore the

317 relationship between RFI annotation sensitivity and $\mathrm{MS}^{2}$ RFI percentage, we split the sampled

$318 \mathrm{MS}^{2}$ spectra into 5 groups according to their RFI count percentages. $\mathrm{MS}^{2}$ spectra with RFI count

319 percentages over $40 \%$ were merged together to ensure that there were enough $\mathrm{MS}^{2}$ spectra for fair

320 comparison. As seen in Figures 6B and 6D, RFI annotation sensitivity does not show general

321 preference for RFI percentage. No statistical significance $(P>0.1$, one-way ANOVA) was

322 observed among the annotation sensitivities of different groups. These results further demonstrate

323 that RFIs in $\mathrm{MS}^{2}$ spectra remain underestimated, and most RFIs in $\mathrm{MS}^{2}$ spectra cannot be correctly

324 identified.

325 


\section{Conclusion}

327 This work provides a comprehensive study of RFIs using large-scale, high-quality, and well328 annotated $\mathrm{MS}^{2}$ spectra data from the NIST $20 \mathrm{MS}$ spectral library. Our results of ion-count and

329 ion-intensity percentages of RFIs suggest that RFIs are common in the CID MS ${ }^{2}$ spectra of 330 different classes of chemicals. The high occurrence of RFIs is well beyond our previous knowledge, 331 which indicates a need for attention during the development of bioinformatic tools for in silico 332 fragmentation as well as de novo $\mathrm{MS}^{2}$ spectra interpretation. More importantly, the in-depth 333 interpretation of RFIs extends our current understanding of the CID fragmentation mechanism and 334 fragmentation pathway. It will also guide the development of more precise bioinformatic tools for

335 the interpretation of $\mathrm{MS}^{2}$ spectra, facilitating unknown chemical identification in MS-based 336 chemical analysis. 


\section{Supporting Information}

339 The Supporting Information is available free of charge.

340 Figure S1. Radical precursor ions $\left(\mathrm{M}^{+\bullet} / \mathrm{M}^{-\bullet}\right)$ in $\mathrm{MS}^{2}$ spectra. Figure S2. Existence of radical 341 precursor ions in positively and negatively ionized NIST $20 \mathrm{MS}^{2}$ spectra. Figure S3. Distribution

342 of the number of annotated fragments in NIST 20. Figure S4. RFI intensity percentage 343 distributions of different superclasses. Figure S5. The sunburst plot of RFI count percentage 344 (medians) in negative ion mode. Figure S6. The sunburst plot of RFI intensity percentage 345 (medians) in positive ion mode. Figure S7. The sunburst plot of RFI intensity percentage (medians) 346 in negative ion mode. Figure S8. Distributions of four patterns of change in RFI count percentage 347 with collision energy in both positive and negative ion modes. Figure S9. A fragmentation 348 pathway example including RFIs. Text S1. Subformula annotation of NIST 20. Text S2. SIRIUS 3494 parameter settings. Table S1. ClassyFire results of unique chemicals in NIST 20 (positive ion 350 mode). Table S2. ClassyFire results of unique chemicals in NIST 20 (negative ion mode). Table 351 S3. 307 CDK chemical substructure bits. Table S4. Ion-count percentage distribution of RFIs and 352 protonated fragment ions in NIST 20 (positive ion mode). Table S5. Ion-count percentage 353 distribution of RFIs and deprotonated fragment ions in NIST 20 (negative ion mode). Table S6.

354 Ion-intensity percentage distribution of RFIs and protonated fragment ions in NIST 20 (positive 355 ion mode). Table S7. Ion-intensity percentage distribution of RFIs and deprotonated fragment ions 356 in NIST 20 (negative ion mode). Table S8. Ion-count percentage medians of RFIs in different 357 compound classes in NIST 20 (positive ion mode). Table S9. Ion-count percentage medians of 358 RFIs in different compound classes in NIST 20 (negative ion mode). Table S10. Ion-intensity 359 percentage medians of RFIs in different compound classes in NIST 20 (positive ion mode). Table 360 S11. Ion- intensity percentage medians of RFIs in different compound classes in NIST 20 (negative 
361 ion mode). Table S12. CDK substructures of unique chemicals in NIST 20 (positive ion mode).

362 Table S13. CDK substructures of unique chemicals in NIST 20 (negative ion mode). Table S14.

363 Statistical analysis results of CDK substructures (positive ion mode). Table S15. Statistical

364 analysis results of CDK substructures (negative ion mode).

365

366 


\section{Acknowledgments}

368 This study was funded by University of British Columbia Start-up Grant (F18-03001), Canada

369 Foundation for Innovation (CFI 38159), New Frontiers in Research Fund/Exploration (NFRFE-

370 2019-00789), and National Science and Engineering Research Council (NSERC) Discovery Grant

371 (RGPIN-2020-04895). We also thank Alisa Hui for proofreading this manuscript.

372

373 


\section{References}

375 1. Sleno, L., Volmer, D.A.: Ion activation methods for tandem mass spectrometry. Journal of mass spectrometry. 39, 1091-1112 (2004)

2. Cooks, R.G.: Special feature: Historical. Collision-induced dissociation: Readings and commentary. Journal of Mass spectrometry. 30, 1215-1221 (1995)

3. Wells, J.M., McLuckey, S.A.: Collision - induced dissociation (CID) of peptides and proteins. Methods in enzymology. 402, 148-185 (2005)

4. Cody, R., Burnier, R., Freiser, B.: Collision-induced dissociation with Fourier transform mass spectrometry. Analytical chemistry. 54, 96-101 (1982)

5. Rebick, C., Levine, R.: Collision induced dissociation: A statistical theory. The Journal of Chemical Physics. 58, 3942-3952 (1973)

6. Chai, Y., Sun, H., Pan, Y., Sun, C.: N-centered odd-electron ions formation from collision-induced dissociation of electrospray ionization generated even-electron ions: single electron transfer via ion/neutral complex in the fragmentation of protonated $\mathrm{N}, \mathrm{N}^{\prime}$-dibenzylpiperazines and protonated N-benzylpiperazines. Journal of the American Society for Mass Spectrometry. 22, 1526-1533 (2011)

7. Rinschen, M.M., Ivanisevic, J., Giera, M., Siuzdak, G.: Identification of bioactive metabolites using activity metabolomics. Nature Reviews Molecular Cell Biology. 20, 353-367 (2019)

8. Quinn, R.A., Melnik, A.V., Vrbanac, A., Fu, T., Patras, K.A., Christy, M.P., Bodai, Z., Belda-Ferre, P., Tripathi, A., Chung, L.K.: Global chemical effects of the microbiome include new bile-acid conjugations. Nature. 579, 123-129 (2020)

9. Xing, S., Jiao, Y., Salehzadeh, M., Soma, K.K., Huan, T.: SteroidXtract: Deep Learning-Based Pattern Recognition Enables Comprehensive and Rapid Extraction of Steroid-Like Metabolic Features for Automated Biology-Driven Metabolomics. Analytical Chemistry. (2021)

10. Böcker, S., Letzel, M.C., Lipták, Z., Pervukhin, A.: SIRIUS: decomposing isotope patterns for metabolite identification. Bioinformatics. 25, 218-224 (2009)

11. Wolf, S., Schmidt, S., Müller-Hannemann, M., Neumann, S.: In silico fragmentation for computer assisted identification of metabolite mass spectra. BMC bioinformatics. 11, 1-12 (2010)

12. Djoumbou Feunang, Y., Eisner, R., Knox, C., Chepelev, L., Hastings, J., Owen, G., Fahy, E., Steinbeck, C., Subramanian, S., Bolton, E., Greiner, R., Wishart, D.S.: ClassyFire: automated chemical classification with a comprehensive, computable taxonomy. Journal of Cheminformatics. 8, 61 (2016)

13. Feunang, Y.D., Eisner, R., Knox, C., Chepelev, L., Hastings, J., Owen, G., Fahy, E., Steinbeck, C., Subramanian, S., Bolton, E.: ClassyFire: automated chemical classification with a comprehensive, computable taxonomy. Journal of cheminformatics. 8, 1-20 (2016)

14. Willighagen, E.L., Mayfield, J.W., Alvarsson, J., Berg, A., Carlsson, L., Jeliazkova, N., Kuhn, S., Pluskal, T., Rojas-Chertó, M., Spjuth, O.: The Chemistry Development Kit (CDK) v2. 0: atom typing, depiction, molecular formulas, and substructure searching. Journal of cheminformatics. 9, 1-19 (2017)

15. Dührkop, K., Fleischauer, M., Ludwig, M., Aksenov, A.A., Melnik, A.V., Meusel, M., Dorrestein, P.C., Rousu, J., Böcker, S.: SIRIUS 4: a rapid tool for turning tandem mass spectra into metabolite structure information. Nature Methods. 16, 299-302 (2019)

16. Rasche, F., Svatoš, A., Maddula, R.K., Böttcher, C., Böcker, S.: Computing Fragmentation Trees from Tandem Mass Spectrometry Data. Analytical Chemistry. 83, 1243-1251 (2011) 
17. Guo, J., Shen, S., Xing, S., Chen, Y., Chen, F., Porter, E.M., Yu, H., Huan, T.: EVA: Evaluation of Metabolic Feature Fidelity Using a Deep Learning Model Trained With Over 25000 Extracted Ion Chromatograms. Analytical Chemistry. (2021)

18. Rasche, F., Scheubert, K., Hufsky, F., Zichner, T., Kai, M., Svatoš, A., Böcker, S.: Identifying the Unknowns by Aligning Fragmentation Trees. Analytical Chemistry. 84, 3417-3426 (2012)

19. Djoumbou-Feunang, Y., Pon, A., Karu, N., Zheng, J., Li, C., Arndt, D., Gautam, M., Allen, F., Wishart, D.S.: CFM-ID 3.0: Significantly improved ESI-MS/MS prediction and compound identification. Metabolites. 9, 72 (2019)

20. Dührkop, K., Shen, H., Meusel, M., Rousu, J., Böcker, S.: Searching molecular structure databases with tandem mass spectra using CSI: FingerID. Proceedings of the National Academy of Sciences. 112, 12580-12585 (2015)

21. Ridder, L., van der Hooft, J.J., Verhoeven, S., de Vos, R.C., Bino, R.J., Vervoort, J.: Automatic chemical structure annotation of an LC-MS $\mathrm{n}$ based metabolic profile from green tea. Analytical Chemistry. 85, 6033-6040 (2013)

22. Ridder, L., van der Hooft, J.J., Verhoeven, S., de Vos, R.C., van Schaik, R., Vervoort, J.: Substructure - based annotation of high - resolution multistage MSn spectral trees. Rapid Communications in Mass Spectrometry. 26, 2461-2471 (2012)

23. Wang, Y., Kora, G., Bowen, B.P., Pan, C.: MIDAS: a database-searching algorithm for metabolite identification in metabolomics. Analytical chemistry. 86, 9496-9503 (2014)

24. Tsugawa, H., Kind, T., Nakabayashi, R., Yukihira, D., Tanaka, W., Cajka, T., Saito, K., Fiehn, O., Arita, M.: Hydrogen rearrangement rules: computational MS/MS fragmentation and structure elucidation using MS-FINDER software. Analytical chemistry. 88, 7946-7958 (2016)

25. Huan, T., Tang, C., Li, R., Shi, Y., Lin, G., Li, L.: MyCompoundID MS/MS search: metabolite identification using a library of predicted fragment-ion-spectra of 383,830 possible human metabolites. Analytical chemistry. 87, 10619-10626 (2015) 


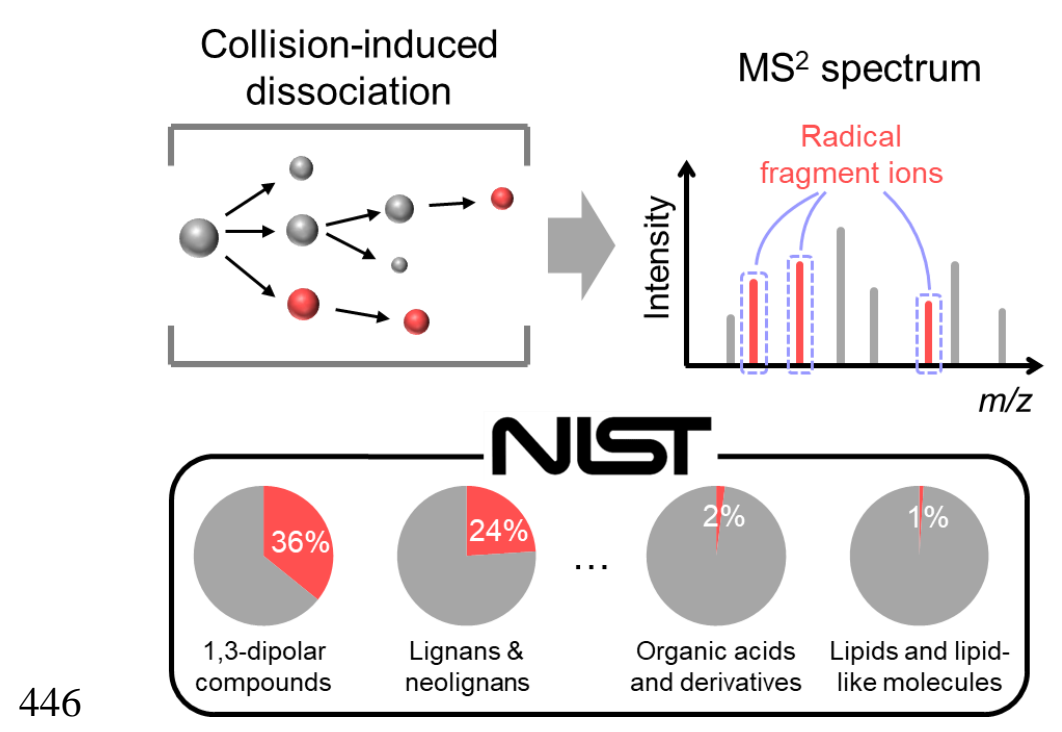

447 


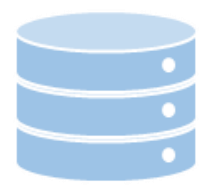

\section{NIST 20}

$\mathrm{MS}^{2}$ with subformula annotation

$\nabla$ Precursor $\mathrm{C}_{12} \mathrm{H}_{12} \mathrm{NOS}_{2}{ }^{+}$

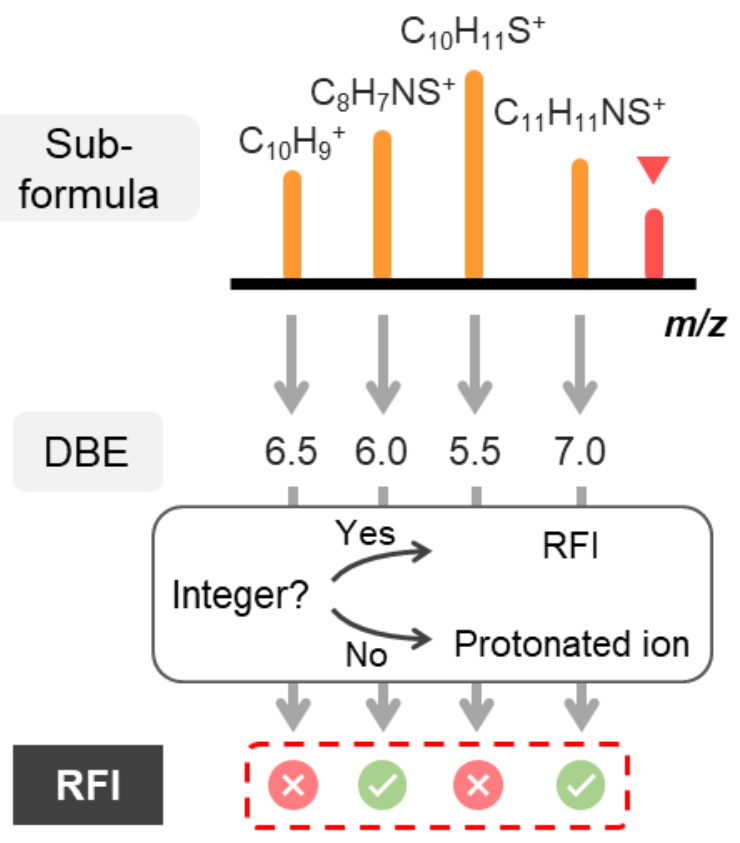

449 Figure 1. Schematic workflow of mining NIST 20 to automatically explore RFIs. 

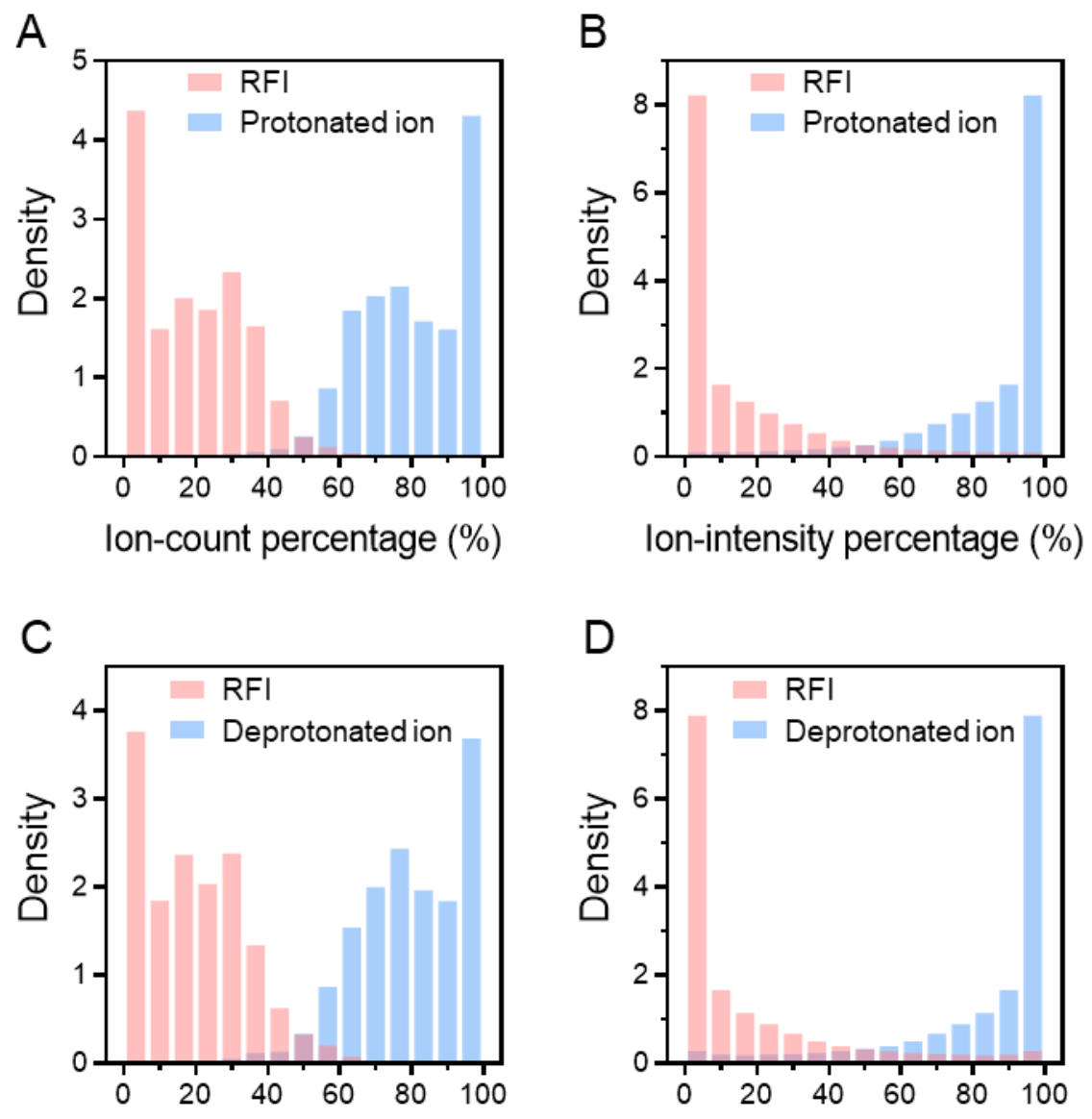

D

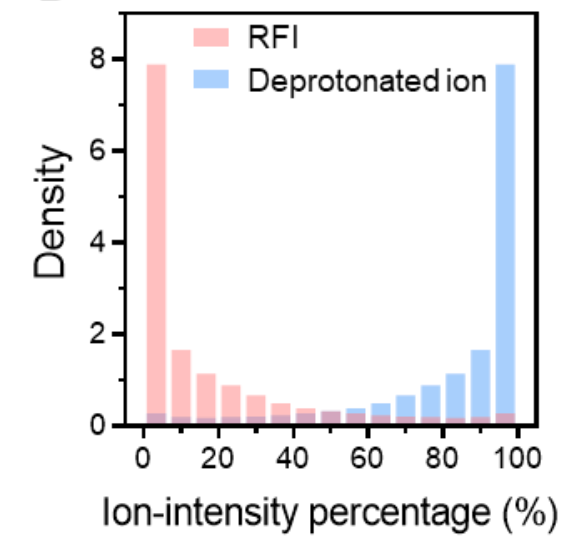

452 Figure 2. Distribution plots of RFIs \& (de)protonated ions in NIST 20 library. (A) \& (C) Ion-

453 count distribution of RFIs and (de)protonated fragment ions. (B) \& (D) Ion-intensity distribution 454 of RFIs and (de)protonated fragment ions. 
A

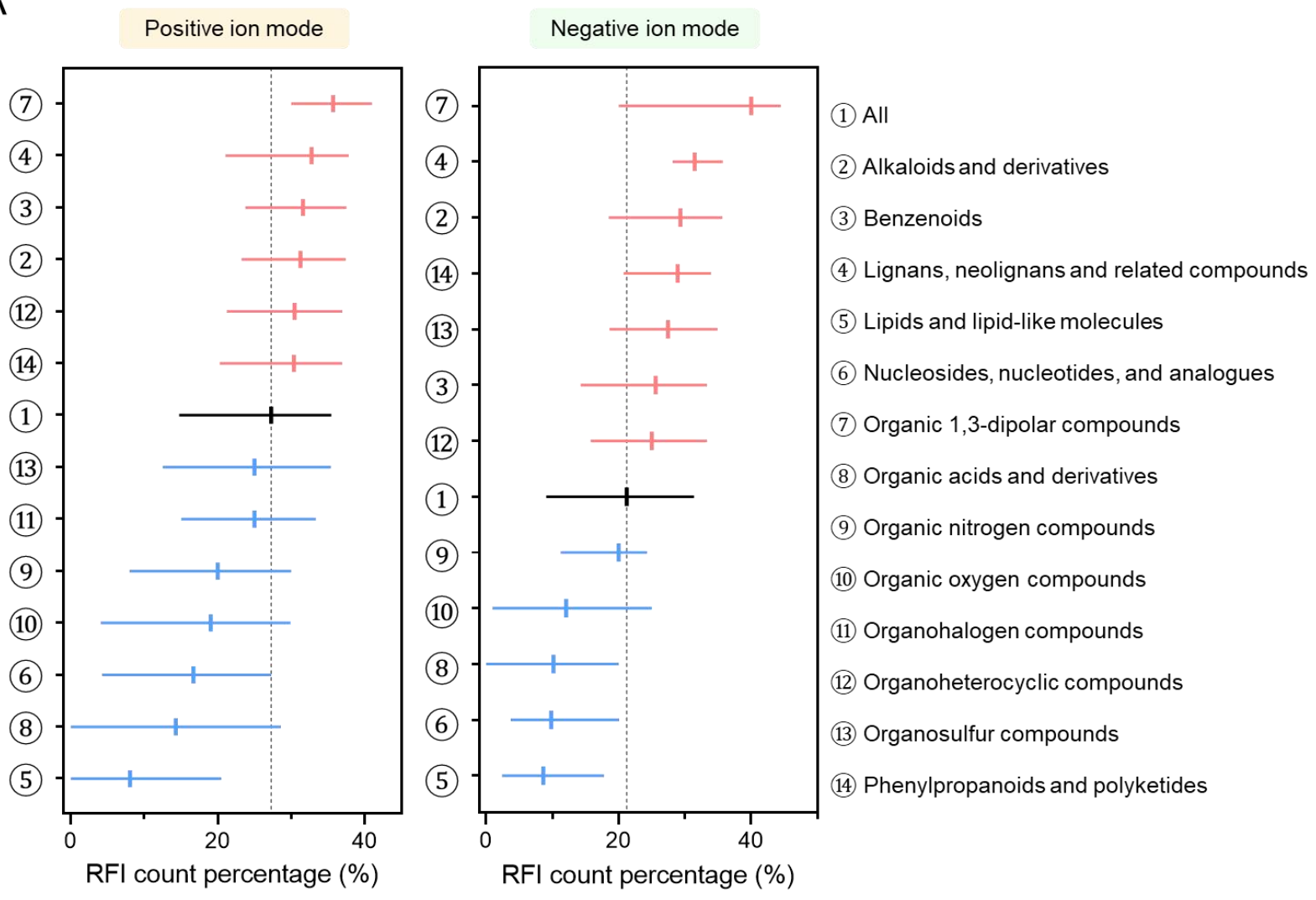

B

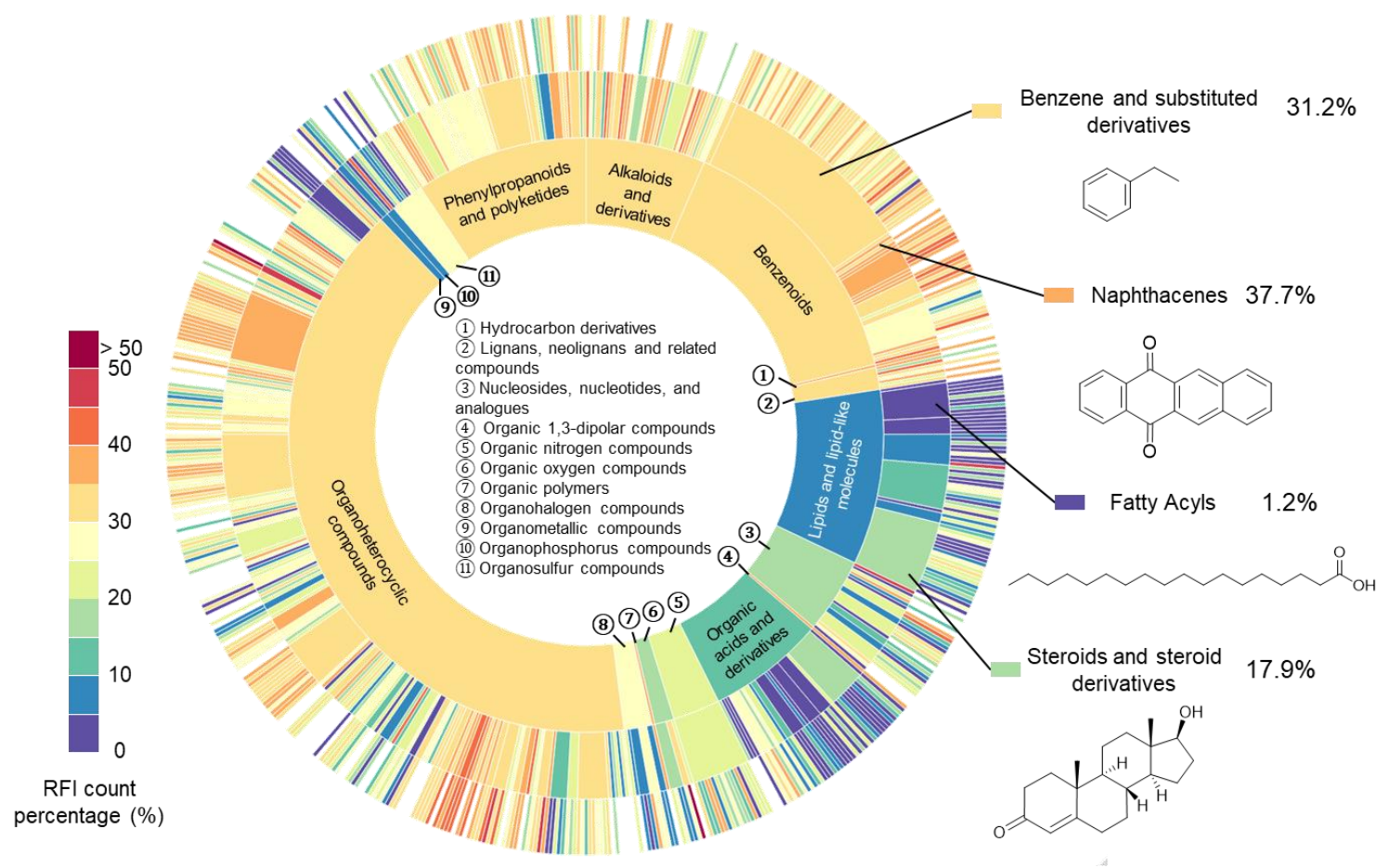


457 Figure 3. (A) RFI count percentages at the level of "Superclass" (median with interquartile range). 458 The box plots were drew using median with interquartile. Compound superclasses containing more 459 than $0.1 \%$ of the total compounds (13 superclasses in each ion mode) are shown. (B) The sunburst 460 plot of RFI count percentage (medians) in positive ion mode. Slices from the inner layer to the 461 outer layer represent class levels of "superclass", "class" and "subclass".

462

463 


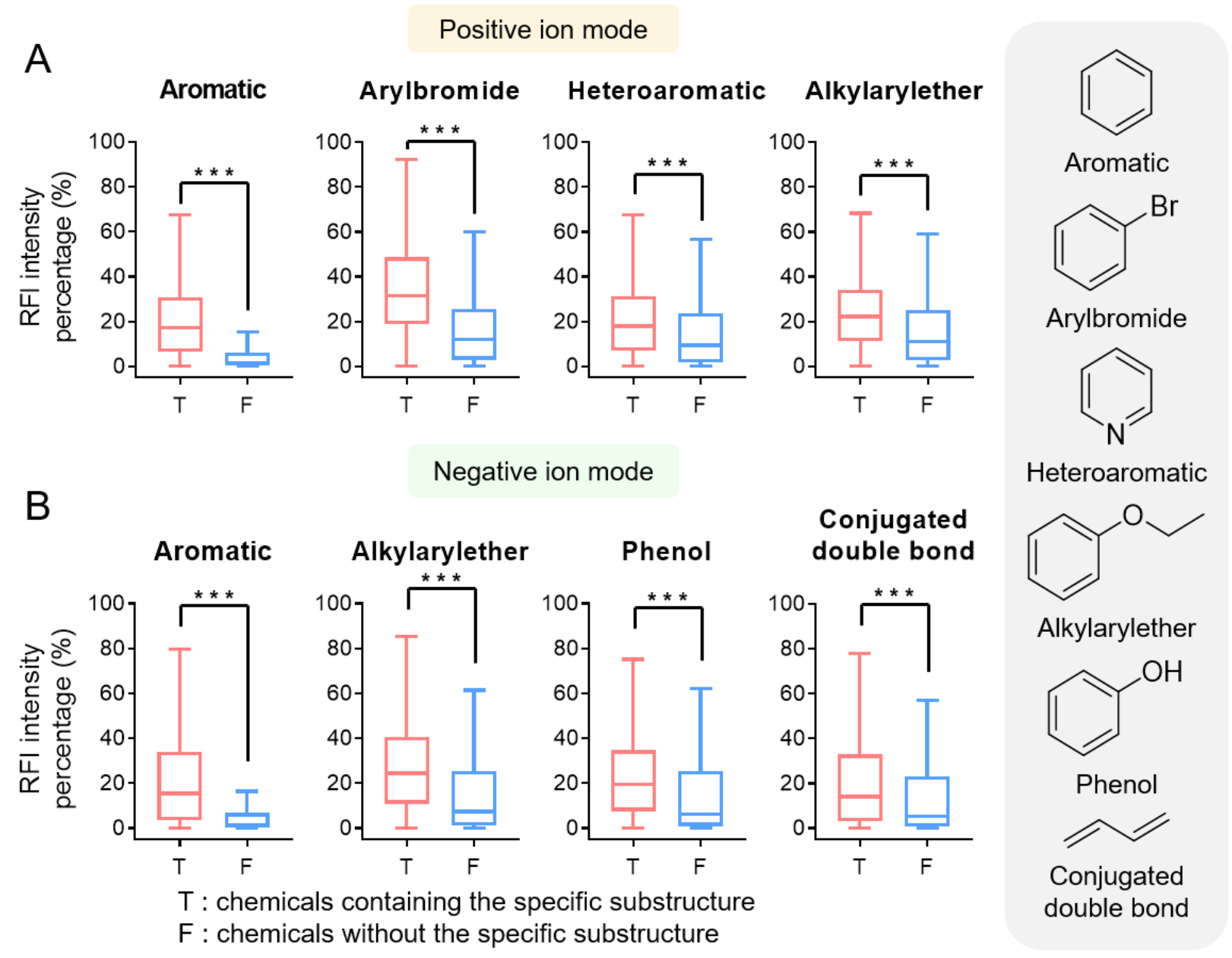

Figure 4. Representative chemical substructures that tend to produce RFIs when a chemical 466 contains it in $(\mathrm{A})$ positive ion mode and $(\mathrm{B})$ negative ion mode. (*** on top of the box plot means $467 \mathrm{p}<0.001$, error bars indicate $95 \%$ confidence interval). 
A

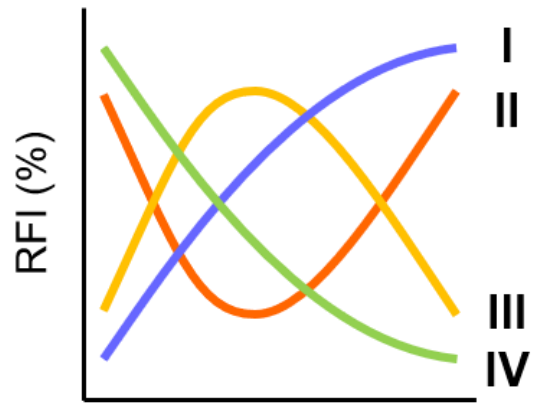

Collision energy

B

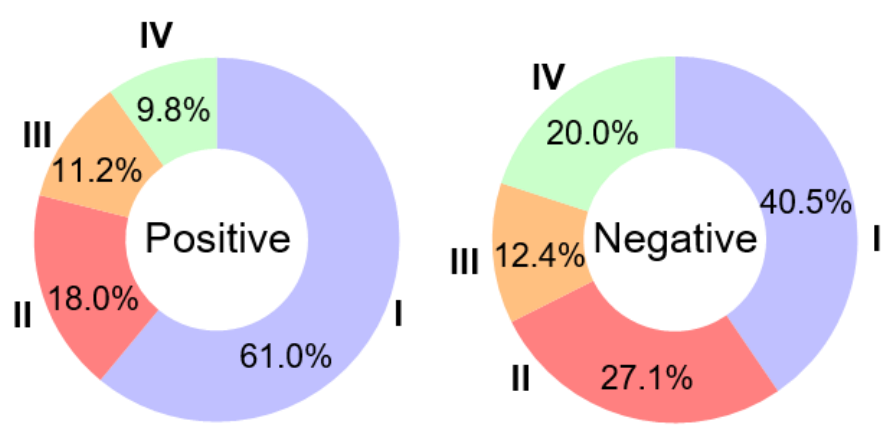

C

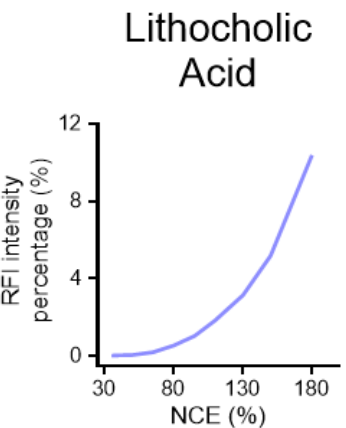

\section{Arachidonic Acid} Biotinamide
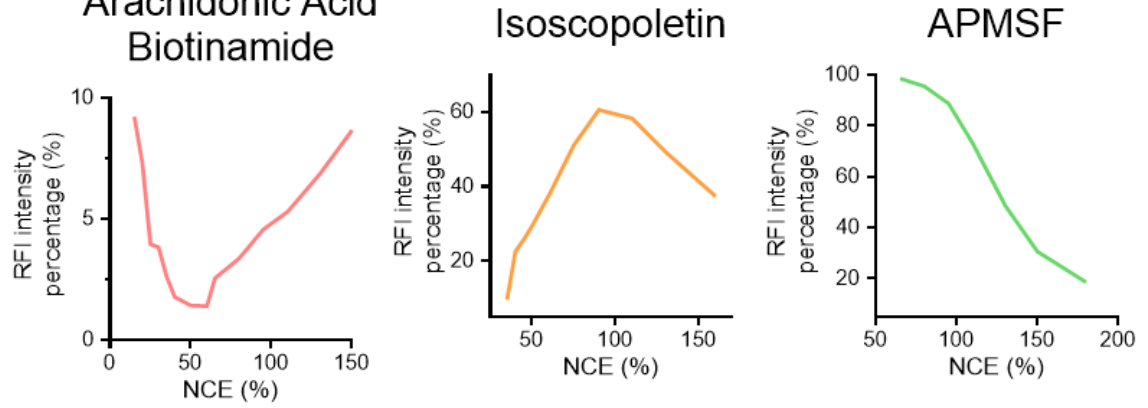

471 Figure 5. (A) Four patterns of how RFI percentage changes with collision energy. (B)

472 Distributions of the four patterns in both positive and negative ion modes. (C) Representative

473 examples of the four patterns. (NCE: normalized collision energy). APMSF: (4-

474 Carbamimidoylphenyl) methanesulfonyl fluoride.

475

476 


\section{Positive ion mode}

A

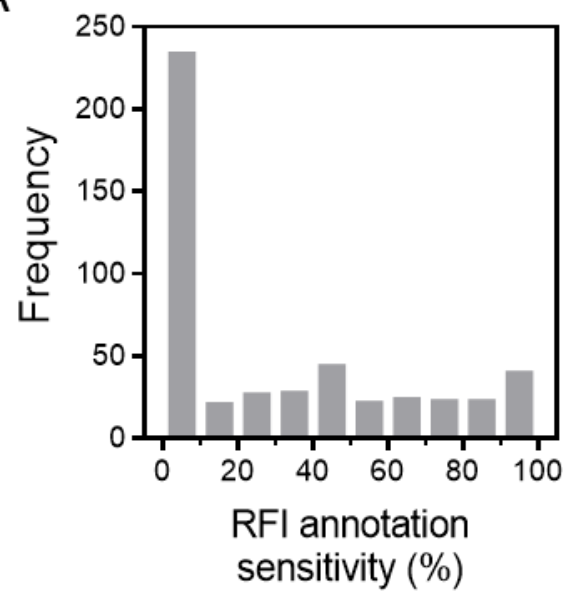

B

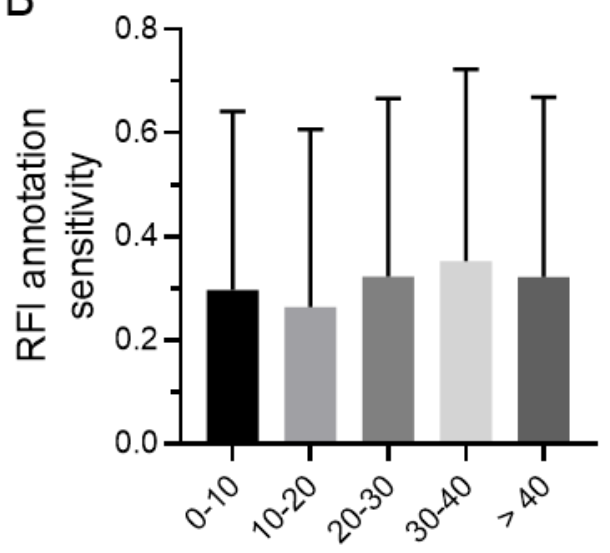

RFI count percentage $(\%)$

Negative ion mode

C

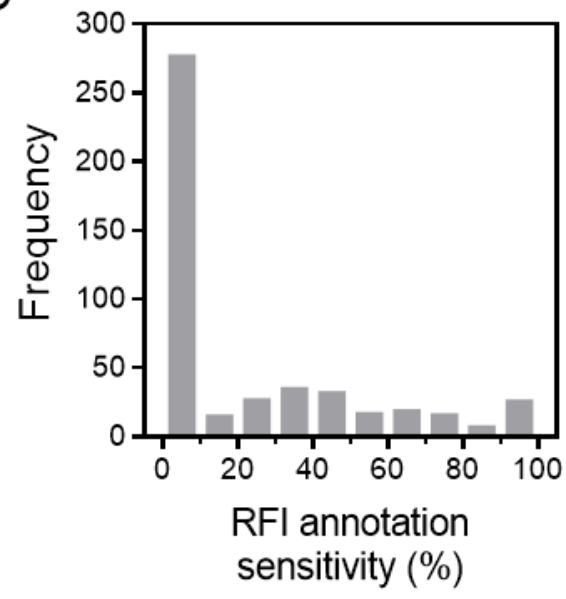

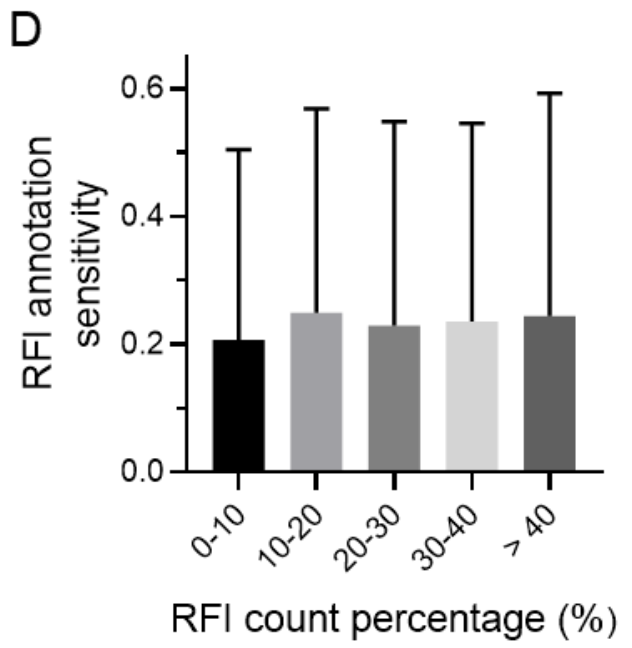

478 Figure 6. RFI annotation results using SIRIUS 4. (A) Distributions of RFI annotation sensitivity

479 in positive ion mode. (B) RFI annotation sensitivity in terms of different RFI count percentages in 480 positive mode (mean with SD shown). (C) Distributions of RFI annotation sensitivity in negative 481 ion mode. (D) RFI annotation sensitivity in terms of different RFI count percentages in negative 482 ion mode (mean with SD shown). 
Table 1. Summary of representative in silico fragmentation tools (in alphabetical order) and their

RFI implementations.

\begin{tabular}{|c|c|c|}
\hline Tool name & $\begin{array}{l}\text { Core algorithm for in silico } \\
\text { fragmentation }\end{array}$ & RFI implementation \\
\hline CFM-ID 3.0[19] & $\begin{array}{l}\text { Models fragmentation as a } \\
\text { stochastic, homogenous, Markov } \\
\text { process involving state transitions } \\
\text { between charged fragments. }\end{array}$ & $\begin{array}{l}\text { No. Even-electron rule is applied, } \\
\text { and no RFI is considered. }\end{array}$ \\
\hline CSI:FingerID[20] & $\begin{array}{l}\text { Computes fragmentation trees of } \\
\mathrm{MS}^{2} \text { spectra. Predicts their } \\
\text { fingerprints and compares them } \\
\text { against the fingerprints of } \\
\text { candidate compounds in the } \\
\text { structure database. }\end{array}$ & $\begin{array}{c}\text { Partially. A few common radical } \\
\text { losses are taken into } \\
\text { consideration. }\end{array}$ \\
\hline MAGMa[21, 22] & $\begin{array}{l}\text { Assigns pre-generated } \\
\text { substructures to the fragment ions } \\
\text { of high-resolution multistage } \mathrm{MS}^{\mathrm{n}} \\
\text { data, and ranks the candidate } \\
\text { molecules. }\end{array}$ & $\begin{array}{l}\text { No. The maximum number of } \\
\text { protons by which the mass is } \\
\text { allowed to differ is set to the } \\
\text { number of broken bonds plus } \\
\text { one. }\end{array}$ \\
\hline MetFrag[11] & $\begin{array}{l}\text { A hybrid rule-based combinatorial } \\
\text { approach. Simulates the } \\
\text { fragmentation via breaking } \\
\text { molecular bonds. }\end{array}$ & $\begin{array}{l}\text { Partially. The matching function } \\
\text { adds or removes a hydrogen to } \\
\text { the fragment mass. A penalty is } \\
\text { given in this case. }\end{array}$ \\
\hline $\operatorname{MIDAS}[23]$ & $\begin{array}{l}\text { A three-level fragmentation tree is } \\
\text { constructed for each chemical } \\
\text { structure. Three charged forms are } \\
\text { considered. }\end{array}$ & $\begin{array}{c}\text { Yes. Fragments in forms of }[\mathrm{F}]^{+} \\
{[\mathrm{F}+\mathrm{H}]^{+}, \text {and }[\mathrm{F}+2 \mathrm{H}]^{+} \text {are }} \\
\text { considered. }\end{array}$ \\
\hline MS-FINDER[24] & $\begin{array}{l}\text { Hydrogen rearrangement during } \\
\text { bond cleavage \& even-electron rule } \\
\text { for carbon and heteroatoms. }\end{array}$ & $\begin{array}{l}\text { Partially. Up to two hydrogens } \\
\text { can be added or removed to } \\
\text { recognize RFIs, and RFIs are } \\
\text { considered as irregular behaviors } \\
\text { (semiresolved). }\end{array}$ \\
\hline MycompoundID[25] & $\begin{array}{l}\text { Heteroatom-initiated bond } \\
\text { chopping \& splitable-bond } \\
\text { chopping }\end{array}$ & $\begin{array}{l}\text { No. Only }[\mathrm{M}+\mathrm{H}]^{+} \text {and }[\mathrm{M}-\mathrm{H}]^{-} \\
\text {are considered. }\end{array}$ \\
\hline
\end{tabular}

